\title{
Microsensor for in situ flow measurements in benthic boundary layers at submillimeter resolution with extremely slow flow
}

\author{
Andreas Brand, ${ }^{1,5}$ Beat Müller, ${ }^{1,5}$ Alfred Wüest, ${ }^{1,5}$ Christian Dinkel, ${ }^{1}$ Niels Peter Revsbech, ${ }^{2}$ Lars Peter Nielsen, ${ }^{2}$ \\ Ole Pedersen, ${ }^{3}$ Lars Riis Damgaard, ${ }^{4}$ Lars Hauer Larsen, ${ }^{4}$ and Bernhard Wehrli, ${ }^{1,5}$ \\ ${ }^{1}$ Swiss Federal Institute of Aquatic Science and Technology, Eawag, CH-6047 Kastanienbaum, Switzerland \\ ${ }^{2}$ University of Aarhus, Institute of Biology, Dept. of Microbiology, Ny Munkegade B. 540, DK-8000 Aarhus C, Denmark \\ ${ }^{3}$ University of Copenhagen, Freshwater Biological Laboratory, Helsingørsgade 51, DK-3400 Hillerød, Denmark \\ ${ }^{4}$ Unisense A/S, Brendstrupgaardsvej 21 F, DK-8200 Aarhus N, Denmark \\ ${ }^{5}$ Institute of Biogeochemistry and Pollutant Dynamics, ETH, CH-8092 Zürich, Switzerland
}

\begin{abstract}
The currents in the lowest few millimeters of the bottom boundary layer of lakes are highly important for the dissipation of kinetic energy and for chemical processes such as oxygen transfer into the sediment. So far, no highresolution flow velocity profiles close to the sediment-water interface have been reported for such systems because a suitable flow meter was lacking. This article introduces a novel sensor for the measurement of extremely lowflow velocities. The sensor is based on a gas transducer surrounded by a gas reservoir. It measures the change in the partial pressure of a tracer gas outside the reservoir tip due to advective transport. The sensor is suitable for measurements of velocities smaller than $1 \mathrm{~mm} \mathrm{~s}^{-1}$, with a spatial resolution of 100 to $250 \mu \mathrm{m}$. The flow measurements prove to be insensitive to temperature changes between 5 and $15{ }^{\circ} \mathrm{C}$. The sensor is robust against relative pressure changes, and angular differences in the sensitivity can be calibrated. We present high-resolution in situ measurements at the bottom of a pre-alpine lake with shear velocities as low as $0.13 \pm 0.02 \mathrm{~cm} \mathrm{~s}^{-1}$. The velocity profile nicely resolves the transition zone between the viscous and the logarithmic boundary layer.
\end{abstract}

\section{Introduction}

The aquatic benthic boundary layer (BBL) is the interface between sediments and the overlying waters in oceans, lakes, wetlands, and rivers (Boudreau and Jørgensen 2001). It is the main location of (i) dissipation of energy of currents and waves and (ii) turbulence and mixing in the stratified interior (Wüest and Lorke, 2003). Below this interface, early diagenetic processes of settled particulate matter cause chemical concentration gradients in the pore water (Boudreau and Jørgensen 2001). Processes on both sides of the interface have been shown to influence the exchange of dissolved substances between the sediment and the overlying water. Whereas the role of the BBL in biogeochemical processes has been extensively investigated (Boudreau and Guinasso 1982; Jørgensen and Revsbech 1985; Jørgensen and Marais 1990; Lorke et al. 2003), studies on properties of currents

\footnotetext{
Acknowledgments

We thank Michael Schurter and Daniela Richter for the help in the field and Daniel McGinnis and Theresa Edmonds who improved the English of the manuscript. This study was supported by the Swiss National Science Foundation (grant no. 200020-103827.1).
}

in the few millimeters immediately above the sediment are scarce (Caldwell and Chriss 1979; Chriss and Caldwell 1982; Chriss and Caldwell 1984a; Chriss and Caldwell 1984b). To our knowledge, Caldwell and Chriss (1979) performed the only in situ measurements in the viscous boundary layer (VBL) in the ocean. No comparable measurements have been reported for lakes, where deep-water flow velocities are much lower than in the ocean, and so far no probe of suitable sensitivity and size has been developed and applied.

A variety of methods exist for the measurement of flows in natural waters close to the sediment (Khalili et al. 2001). One of the more widely used methods is laser Doppler anemometry, which measures with high precision and fast response times, making it suitable for the characterization of vortices and turbulent environments (Nelson et al. 1995). In situ applications have a typical spatial resolution in the range of several millimeters (Agrawal and Belting 1988). Hot film anemometry and hot wire anemometry (HWA) have also been applied, e.g., in studies of shear stress and flow profiles over surfaces in oceanic systems (Gust 1988). Caldwell and Chriss (1979) were able to investigate the viscous boundary layer on the continental shelf 
A

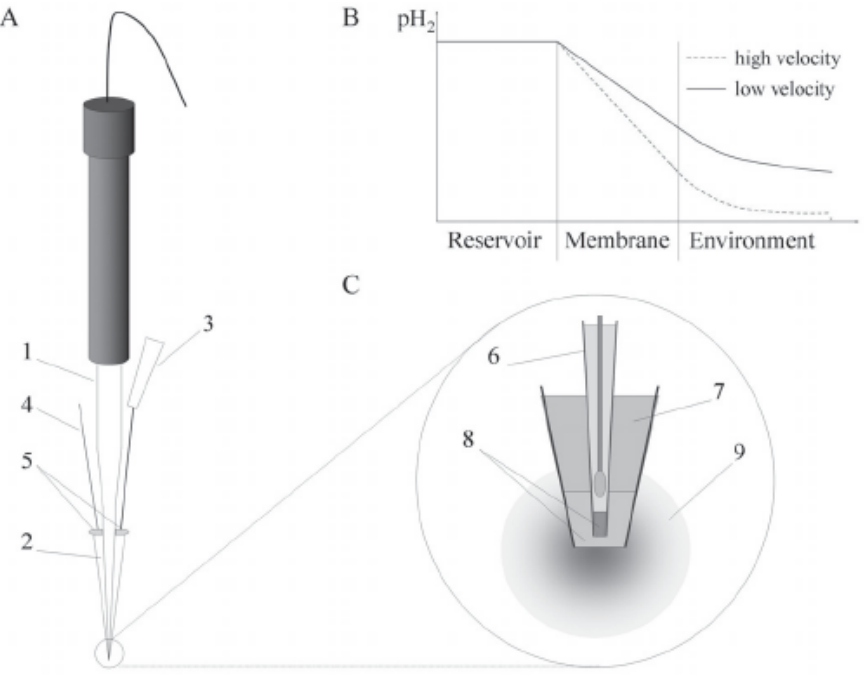

Fig. 1. (A) Overview of the main parts of the sensor. The tracer gas reservoir (2) is flushed through the steel in- and outlets ( 3 and 4). The gas reservoir is situated around the gas transducer (1) and sealed with epoxy resin (5). (B) Concentration profiles in the setup at 2 different flow velocities. A constant partial pressure is applied in the reservoir. At steady state, tracer gas diffuses through the membrane with a constant flux into the environment. The concentration outside the tip is high at low flow (solid curve). If the flow velocity increases, the diffusion sphere is eroded more efficiently and the hydrogen concentration decreases (dashed line). (C) Detailed sketch of the tip. The tracer gas diffuses from the reservoir (7) through the silicone membrane (8) and forms a diffusion sphere (9) around the sensor. Concentration changes are measured in the membrane by the transducer (6).

at $185 \mathrm{~m}$ depth using a heated thermistor. These probes are sturdy and have a fast response, but owing to the induced thermal convection, their detection limit is in the range of $1 \mathrm{~cm} \mathrm{~s}^{-1}$ (Khalili et al. 2001). Very slow flow velocities of $<1 \mathrm{~mm} \mathrm{~s}^{-1}$, however, can be observed by particle image velocimetry (PIV). This method has recently been used to describe the flow in diffusive boundary layers above sediments in the laboratory (Røy et al. 2002) and in the coastal ocean (Bertucciolli et al. 1999, Nimmo Smith et al. 2002; Nimmo Smith et al. 2005). Nevertheless, using PIV in situ demands a very sophisticated experimental setup, and in situ measurements in the lowest region of the VBL have not yet been reported.

To study low velocities in the BBL of lakes, we have developed and applied a new type of miniaturized flow sensor with an extremely low detection limit and a fine spatial resolution for in situ application on a benthic bottom lander. The detection limit is in the submillimeter range, since the probe does not induce any convective currents. It can be used with many existing lander systems equipped with amperometric channels. The novel flow velocity microsensor is based on the principle of the diffusivity sensor presented by Revsbech et al. (1998). The design and principle of the sensor are illustrated in Fig. 1A. Tracer gas is continuously diffusing through the membrane of the reservoir, and a sphere of tracer gas builds up at the tip of the microsensor (Fig. 1C). At steady state, the diffusive loss of tracer gas leads to a linear decrease in partial pressure across the membrane reservoir. In stagnant fluids, the partial pressure gradient builds up, causing a high signal from the gas transducer, whereas high flow rates in the fluids erode the gas sphere (Fig. 1B). This erosion decreases the partial pressure of the tracer at the sensing tip, thereby resulting in low signals from the gas transducer. In our case, we used hydrogen as a tracer gas and a Clark type sensor as the transducer, which measures a current caused by the reduction of hydrogen at the anode. The maximum reduction current is therefore measured under stagnant conditions. The tracer must be inert within the measurement volume but may be any gas that the transducer is able to detect.

\section{Materials and Procedures}

Sensor design-We applied hydrogen as a tracer gas and a hydrogen microsensor (Unisense A/S) as a transducer within the flow sensor (Fig. 1A). Otherwise, it was constructed as described by Revsbech et al. (1998), except that the tip diameters were 40 to $50 \mu \mathrm{m}$ and thus considerably smaller than the typical diffusivity sensors they described. The 2 thin steel capillaries in contact with the otherwise sealed hydrogen reservoir allowed a constant flushing with hydrogen gas. The outflow capillary had a much smaller diameter than the inflow capillary so that the pressure within the reservoir at high gas flows almost corresponded to the pressure within the inlet line.

Sensor calibration-We used the setup shown in Fig. 2 for all calibrations and tests of the sensor in the laboratory. A polyurethane disc (1) with a water-filled $4 \mathrm{~mm}$ wide and $10 \mathrm{~mm}$ deep groove with a radius of $51 \mathrm{~mm}$ was mounted on a modified peristaltic pump (2) (Gilson Minipulse). The disc was kept in a perspex container under water-saturated atmosphere to

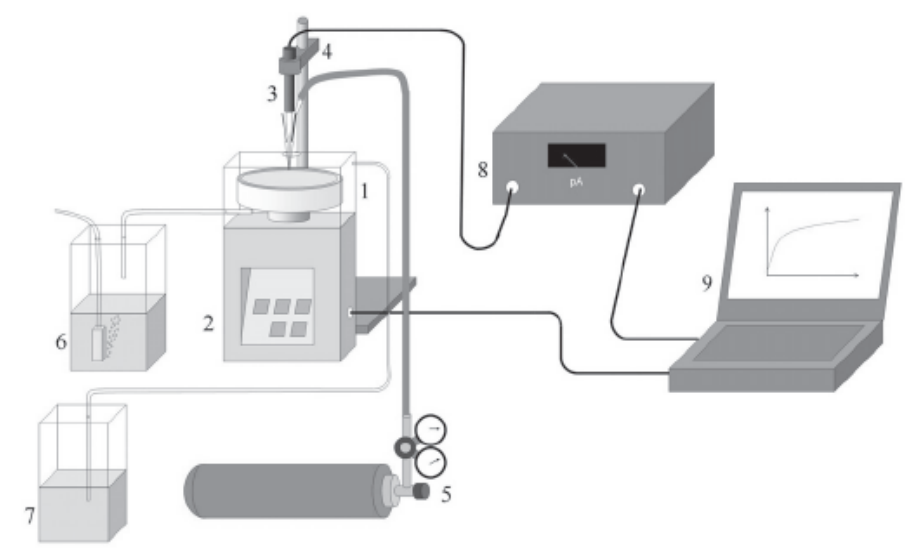

Fig. 2. Setup for the sensor calibration. A plastic disc (1) with a groove is mounted on a modified hosepump (2). The sensor (3) is fixed in a lab stand (4) and inserted into the water-filled groove. The gas inlet of the sensor is connected to a gas bottle with a standard pressure reducer (5). The disc is contained in a box flushed with water-saturated air produced by bubbling air through water in the plastic container (6). The reduction current of the sensor is recorded using a picoamperemeter (8). The hosepump and data acquisition are controlled automatically. 
avoid currents induced by air draught and thermal convection due to evaporative cooling of the water surface. The air was moisturized by bubbling air through water in a container (6). The sensor was then positioned in the water-filled groove using horizontal and vertical micromanipulators. Hydrogen gas flow was controlled with a standard pressure reducer for outlet pressures of up to $1 \mathrm{MPa}$. The sensor signal was recorded with a commercially available picoamperemeter (UNISENSE PA 2000) after A/D conversion (ADC 100 A/D converter). Data acquisition, evaluation, and remote controlling of the peristaltic pump were performed using LabVIEW scripts.

Before each calibration the groove of the disc was filled with fresh water. After filling completely, a small portion of the water was removed until the curvature of the meniscus in the groove remained constant. The sensor was inserted $2 \mathrm{~mm}$ deep into the water and allowed to equilibrate for $10 \mathrm{~min}$ under stagnant conditions. Calibrations were performed in the range of $14 \mathrm{~mm} \mathrm{~s}^{-1}$ down to $0.2 \mathrm{~mm} \mathrm{~s}^{-1}$. Velocities were regulated by setting the hose pump to the corresponding rotation speed. The motor speed stability of the pump is $0.5 \%$. Each interval of constant speed was recorded for $60 \mathrm{~s}$, and an average of the last $15 \mathrm{~s}$ was used to calculate calibration curves. The response was calculated as the ratio of maximum reduction current at $u=0 \mathrm{~mm} \mathrm{~s}^{-1}$ and the actual current.

Sensor performance-Calibrations were performed in the temperature range of 5 to $22{ }^{\circ} \mathrm{C}$ at a reservoir pressure of $0.2 \mathrm{MPa}$ to investigate the influence of temperature on the sensor response. The whole setup was operated in a climate chamber which was sequentially working at $5,7,10,15$, and $22^{\circ} \mathrm{C}$. The system was allowed to adapt completely to the new temperature for at least $5 \mathrm{~h}$ before the sensor calibration procedure was applied. The influence of pressure variation on the signal was investigated by performing standard calibrations under 0.2 , $0.3,0.4$, and $0.5 \mathrm{MPa}$ pressure in the hydrogen reservoir. Two different methods were used to determine the response times of the sensors. In a first attempt, 4 different flow velocities were applied after stagnant conditions. After that, the flow was stepwise decelerated from $1.8 \mathrm{~mm} \mathrm{~s}^{-1}$ to $0.2 \mathrm{~mm} \mathrm{~s}^{-1}$ and then accelerated stepwise again. To determine the sensitivity of the sensor signals to flow direction, standard calibrations were performed for different orientations of the sensor. One position of the sensor was arbitrarily marked as the zero orientation. Successive calibrations were performed every 15 degrees.

In-situ measurements-In situ measurements were carried out in May 2005 in Lake Alpnach, a medium-sized mesotrophic sub-basin of Lake Lucerne in central Switzerland. It has an elliptical shape of approximately 5 by $1.5 \mathrm{~km}$, a surface area of $4.2 \mathrm{~km}^{2}$, and a maximum depth of $34 \mathrm{~m}$. The lake is well known for its persistent basin-scale deep-water seiching of several $\mathrm{cm} \mathrm{s}^{-1}$ amplitude with a period of more than $8 \mathrm{~h}$ in length (Gloor et al. 1994; Lorke et al. 2003), which results from the lake's physical dimensions, stratification, and regular wind forcing.

To conduct field measurements, we expanded our benthic lander system LISA (Müller et al. 2002) with an amperometric

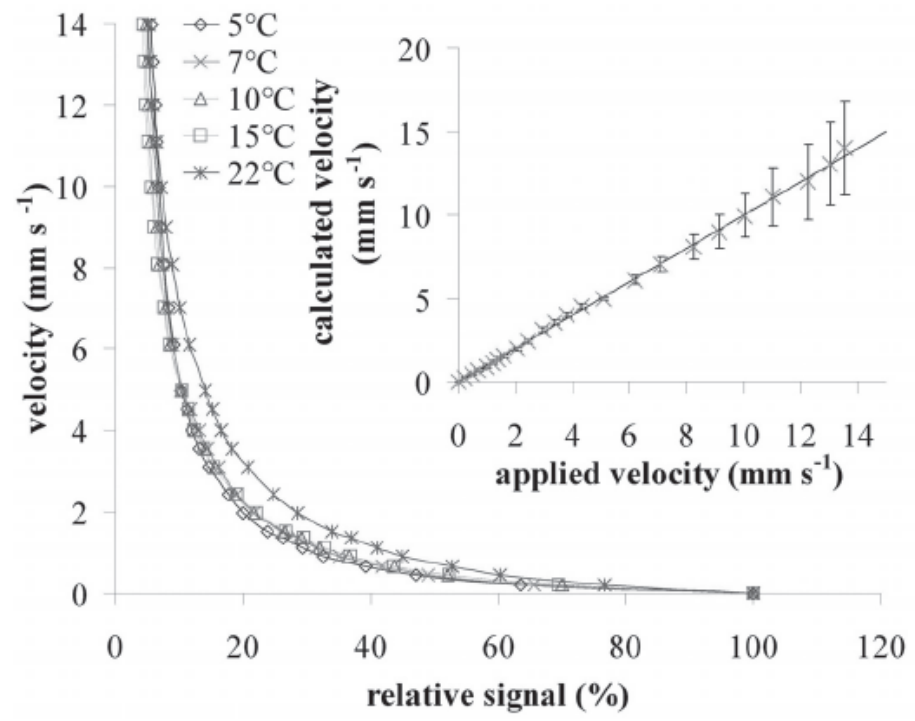

Fig. 3. Calibration curves of a sensor at different temperatures. The inset compares the applied velocities to the velocities calculated from the calibration at $7{ }^{\circ} \mathrm{C}$. Error bars indicate the deviations estimated from the sensitivity of the calibration curve and the standard deviation calculated from the signals recorded at $5,7,10$, and $15^{\circ} \mathrm{C}$.

channel for the flow sensor. A compass was built into LISA to determine the sensor orientation. A 3.5-L hydrogen tank with a high-precision pressure reducer (Tescom) was used as a hydrogen supply. The pressure reducer was adjusted for a constant pressure difference of $0.15 \mathrm{MPa}$, which resulted in a reservoir pressure of $0.45 \mathrm{MPa}$ at the investigated location in $30 \mathrm{~m}$ depth. The sensor was calibrated before each measurement campaign using water from the investigated lake at $7{ }^{\circ} \mathrm{C}$. Profiles were recorded starting at approximately $1.5 \mathrm{~cm}$ above the sediment, which consisted of fine silty material. A time series of $120 \mathrm{~s}$ was recorded at $3 \mathrm{~Hz}$ at each depth. The first $30 \mathrm{~s}$ were omitted in the data evaluation to avoid artifacts caused by the sensor response time. Thus, averages were calculated in the time interval of 30 to $120 \mathrm{~s}$. For comparison of flow direction and velocities, an acoustic Doppler velocity meter (ADV; Nortek) was installed stationary $11 \mathrm{~cm}$ above the sediment approximately $10 \mathrm{~m}$ apart from LISA. All errors for the values calculated by regression were estimated using the methods described in Bronstein et al. (1997) at a probability value of 0.1 . Linear error propagation was used to estimate uncertainty of interpolated and extrapolated variables.

\section{Assessment}

Sensor performance-Fig. 3 shows typical calibration curves at temperatures between 5 and $22{ }^{\circ} \mathrm{C}$. We approximated the velocity $u[\mathrm{~mm} / \mathrm{s}]$ using the empirical function

$$
u(S)=A * \exp (k *(S-B))+C * S^{D}-E
$$

of the relative signal $S$ [\%] (Fig. 3) with $A, B, C, D, E$, and $k$ as fit parameters. We chose this function for its flexible structure 


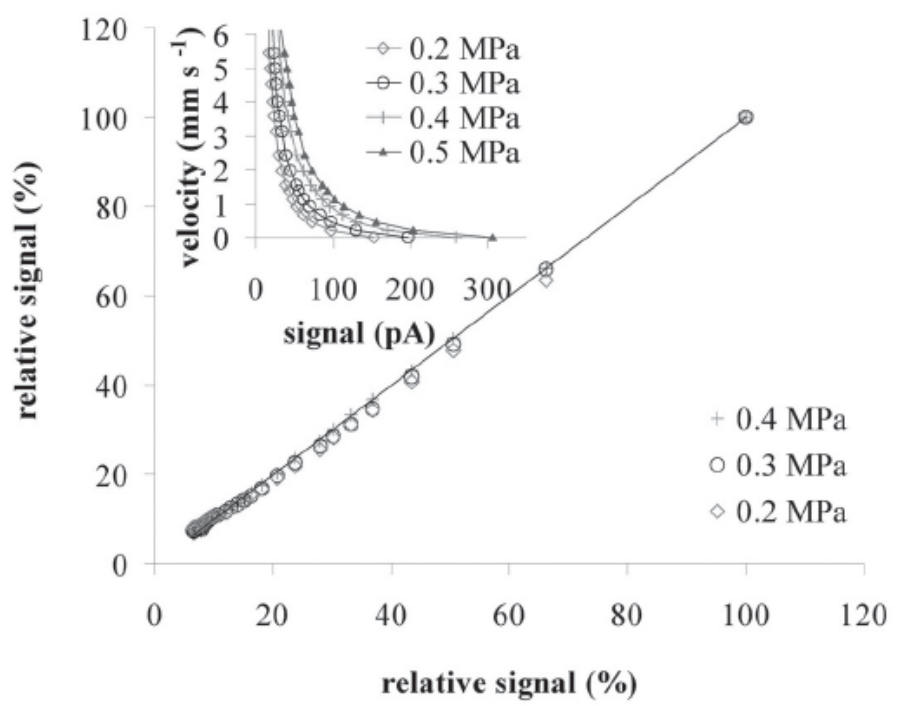

Fig. 4. Influence of reservoir pressure on the signals. The relative signals recorded at different reservoir pressures are compared with those recorded at $0.5 \mathrm{MPa}$. The solid line represents the 1:1 relation. Although the absolute signals increase with increasing pressure (inset), the relative signals agree very well with each other.

and because the observed calibration values are very well approximated. Eq. 1 is not based on physical reasoning, and many different combinations of parameter values describe the observed data with the same quality.

The relative signals of the calibrations between 5 and $15^{\circ} \mathrm{C}$ were almost identical. A substantial difference in the calibration was observed only at $22{ }^{\circ} \mathrm{C}$. In the following analysis of the method's accuracy, we use the data obtained at 5 to $15^{\circ} \mathrm{C}$. The inset of Fig. 3 shows the effect of the difference in the signals on the velocities. Eq. 1 was fitted to the results recorded at $7{ }^{\circ} \mathrm{C}$. The actual velocities are in good agreement with the calculated ones. The error bars show the uncertainty of the velocities $\Delta u$ estimated by

$$
\Delta u=\frac{d u(S)}{d S} * \sigma_{S}
$$

using the local sensitivity of the calibration function $d u(S) / d S$ and the standard deviation $\sigma_{s}$ of the relative signals recorded between 5 and $15{ }^{\circ} \mathrm{C}$. The error is small for slow flow, but at a velocity of $9 \mathrm{~mm} \mathrm{~s}^{-1}$ the relative error exceeds $10 \%$. Owing to the decreasing sensitivity of the sensor for increasing velocities, the uncertainty increases with the flow.

A calibration performed at a reservoir pressure of $0.5 \mathrm{MPa}$ is compared with the calibrations performed at $0.4,0.3$, and $0.2 \mathrm{MPa}$. Fig. 4 shows that, although the absolute signals differ from each other (see inset), the relative signals are close to the $1: 1$ line, indicating almost identical signals. Thus, a calibration recorded in the laboratory for a reservoir pressure of, e.g., 0.2 MPa can be used for in situ measurements at up to $30 \mathrm{~m}$ depth since the relative sensor signal does not depend on the pressure in the reservoir for at least up to $0.5 \mathrm{MPa}$.
Both tests show that the fitting parameters are independent of pressure and temperature in the range relevant for our in situ measurements. The calibration curve varies only between sensors. The $90 \%$ response time varied from 8 to $15 \mathrm{~s}$, depending on the individual sensor.

The spatial resolution of the sensor is not well defined, since it depends on the size of the diffusion sphere of the tracer gas, which is affected by the flow of the surrounding medium. However, a rough estimation can be performed based on the size of the tracer gas sphere under stagnant conditions. According to Crank (1983), the steady-state concentration $C(r)$ at a given distance $r$ of such a gas sphere can be calculated as

$$
C(r)=C_{\infty}+\left(C_{R}-C_{\infty}\right) * R / r^{\prime}
$$

assuming that the tracer is diffusing from a sphere equal to that of the reservoir membrane with a radius $R$. $C_{\infty}$ is the tracer concentration at infinite distance (in our case, $C_{\infty}=0$ ). The radius $r$, where the concentration is still 10\% of the maximum concentration at the sensor tip, is 10 times the tip radius, which is typically 7.5 to $15 \mu \mathrm{m}$. Consequently, $r$ is between 75 and $150 \mu \mathrm{m}$ depending on the size of the sensor. Nevertheless, the size of the diffusion sphere decreases dramatically at the presence of even small flow velocities in the surrounding media, and spatial resolution can be assumed to be much higher if the water is flowing. Therefore we recommend the use of Eq. 3 as a worst-case estimate.

Angular dependence-The dependence on angle ( $\alpha$ ) of the flow sensor signal $S(u, \alpha)$ followed an empirical sinusoidal relationship

$$
S(u, \alpha)=O(u)+\left(m^{*} u+t\right)^{*} \sin (\alpha+\beta) .
$$

where $m, t$, and $\beta$ are fit parameters independent of $u$. Fig. 5 compares experimental sensor signals to functions of Eq. 4,

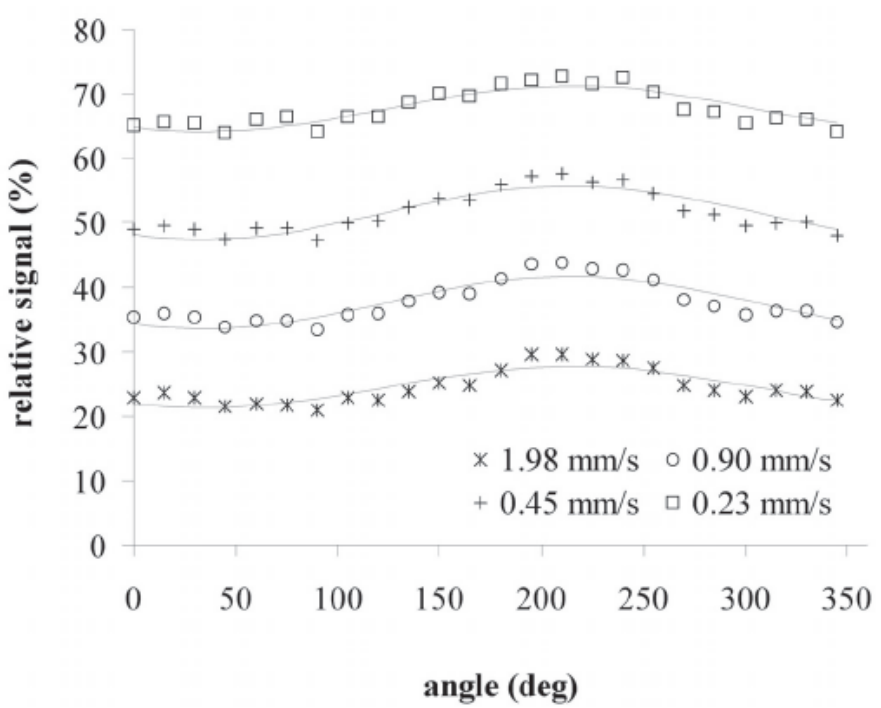

Fig. 5. Angular dependence of the sensor. Relative signals of the flow velocities were recorded at different current angles. The lines represent fitted sinusoidal functions (Eq. 4). 
which were fitted to the measured signals. As is obvious from Fig. 5, the fit parameter $O(u)$ depends on the velocity.

Because we have a formal description of the signal dependence on flow direction (second term in Eq. 4) and a unidirectional calibration curve $\mathrm{O}(u)$ (first term in Eq. 4), we can calculate the velocity in any given situation as long as we know the flow direction $\alpha$ :

For the special case of $\alpha=0$, Eq. 4 reads

$$
S(u, 0)=O(u)+(m * u+t) * \sin (\beta) .
$$

By combining Eqs. 4 and 5, the $\alpha$-independent term $O(u)$ is eliminated by

$$
S(u, 0)=S(u, \alpha) \quad\left(m^{*} u+t\right) *(\sin (\alpha+\beta) \quad \sin (\beta)) .
$$

This elimination permits us to reduce the amount of velocity steps significantly when we examine the direction dependence of the sensor. Otherwise we would have to determine $O(u)$ for every flow velocity. To calculate the flow, we use the calibration performed at a current direction of $\alpha=0$ :

$$
u=B * \exp (k *(S(u, 0) \quad A))+C * S(u, 0)^{D} \quad E .
$$

The two unknown variables $S(u, 0)$ and the flow $u$ can be calculated iteratively using Eqs. 6 and 7.

The parameters of the calibration curve and the angle dependence are determined from the characterization of the sensor in the laboratory. The flow direction $\alpha$ has to be determined from an additional measurement (see below). The flow sensor measurement will give us a signal $S(u, \alpha)$; this value is used in Eq. 7 as an initial estimate for $S(u, O)$. The resulting $u$ is used in Eq. 6 to calculate an improved estimate of $S(u, O)$. This process is repeated until the difference in the successively calculated velocities $u$ changes less than a given value (here $10^{-7} \mathrm{~mm} / \mathrm{s}$ ).

In-situ measurements in Lake Alpnach-The inset of Fig. 6 shows the behavior of the raw signal when the sensor crosses the sediment water interface (SWI). It increases strongly as the sensor approaches the sediment. Because the thickness of a Brinkmann layer in the silty sediment is less than $10 \mu \mathrm{m}$ (Dade et al. 2001) it can be neglected, and the velocity can be assumed to be zero at the SWI. The significantly slower increase in the signal as the sensor penetrates the sediment can be attributed to the decreasing porosity and diffusivity with depth (Revsbech et. al. 1998). The SWI is identified as the position where the kink is observed in the profile. The absolute signal at this position was used as the reference for the calculation of relative signals.

Fig. 6 shows a typical velocity profile recorded during the field campaign. The average horizontal velocities are plotted as a function of the distance from the sediment. The velocity fluctuations are described by the standard deviation of horizontal velocities and are represented by error bars. The transition from the linear zone in the VBL (squares) to the logarithmic layer above (diamonds) (Dade et al. 2001) is reflected in the flow profile. The increase in velocity fluctuations with

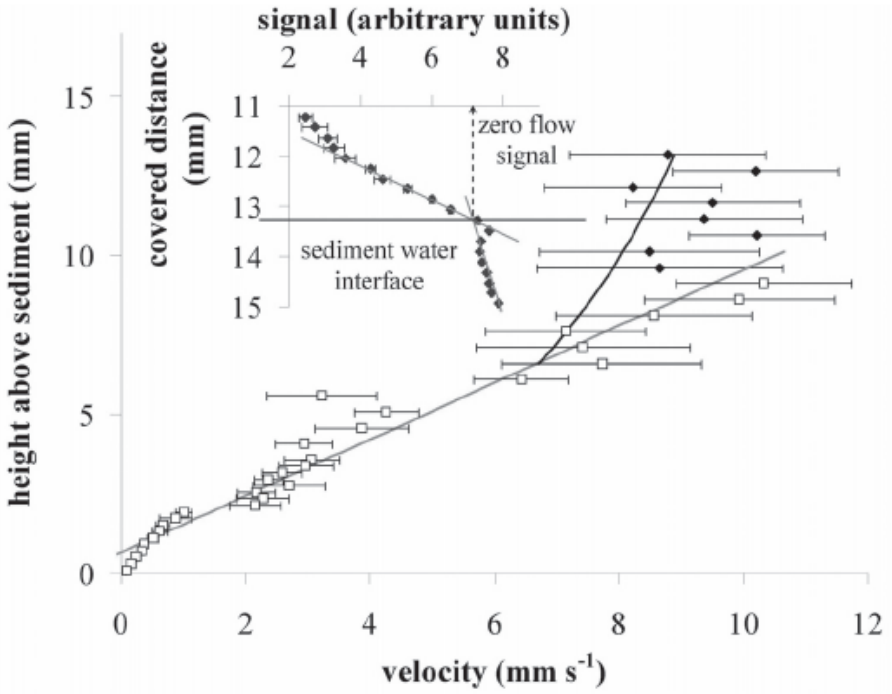

Fig. 6. In situ profile recorded in Lake Alpnach on 13 May 2005. It reflects the transition between the linear boundary layer (squares) and the logarithmic boundary layer (diamonds) at approximately $8 \mathrm{~mm}$ above the sediment. The bars represent the standard deviations of the velocity due to flow fluctuations during the 90 -s recording time and do not reflect measurement errors. The inset shows a detail of the raw data plotted against the distance covered by the sensor. The sediment surface was detected as the point where the kink in the signal was observed. The signal value at the kink was taken as the zero flow reading.

height also reflects the expected behavior of flow at the transition between the VBL and the logarithmic region.

The gray solid line shows the linear fit to the data in the VBL. It is obvious that this region between the SWI and 7.5 $\mathrm{mm}$ above the sediment follows the linear form expected in the VBL. The shear stress $\tau$ in the VBL

$$
\tau=\rho v \frac{d u}{d z}
$$

with the water density $\rho$ and the temperature-dependent kinematic viscosity $v$ [e.g., Caldwell and Chriss (1979)] can be calculated to $1.70 \pm 0.0210^{-3} \mathrm{~N} \mathrm{~m}^{-2}$. The shear velocity $u_{*}$ is defined as

$$
u_{*}=\sqrt{\tau / \rho},
$$

which corresponds to $0.13 \pm 0.01 \mathrm{~cm} \mathrm{~s}^{-1}$. This value was used to calculate the logarithmic profile (dark line),

$$
u(z)=\frac{u_{*}}{\kappa} \ln \left(\frac{z}{z_{0}}\right) .
$$

The roughness parameter $z_{o}$ was taken from the intercept of the linear fit as $z_{o}=0.6 \pm 0.2 \mathrm{~mm}$. We used the usual value of the von Karman constant $\kappa$ of 0.41 (e.g., Chriss and Caldwell 1984b).

To compare the ADV measurements with the results of the flow sensor, we extrapolated the velocity profile as described by the law-of-the-wall (Eq. 10) to the height of the measurement volume of the ADV $11 \mathrm{~cm}$ above the sediment. The calculated velocity of $1.7 \pm 0.1 \mathrm{~cm} \mathrm{~s}^{-1}$ is in excellent agreement with the measured velocity of $1.6 \pm 0.1 \mathrm{~cm} \mathrm{~s}^{-1}$ considering the fact that we are extrapolating over the range of $10 \mathrm{~cm}$. 
The flow sensor works properly only if vertical flows are negligible. The ADV measurements reveal that less than 10\% of the recorded vertical velocities exceeded one-fifth of the horizontal velocity $11 \mathrm{~cm}$ above the sediment. Because we can assume that vertical velocities are even more dampened close to the sediment surface, their influence on the flow sensor signal can be safely ignored.

The current direction was relatively constant, with a standard deviation of \pm 7 degrees during profile recording. Therefore, we can exclude the existence of depth-dependent flow directions in the investigated scale and conclude that the estimation of the flow direction using the ADV $11 \mathrm{~cm}$ above the sediment water interface is sufficient in our case.

\section{Discussion}

The new flow sensor was characterized in the laboratory and tested for its applicability at $27 \mathrm{~m}$ depth in a lake with very low flow at the sediment-water interface. Owing to its high sensitivity at low currents and fine spatial resolution, the sensor provides an excellent tool for the study of very slow flow in the ultimate proximity to sediment boundaries of natural water bodies with extremely weak shear. This instrument fills the gap left by other methods like ADV (low spatial resolution) and HWA (higher speed is necessary).

The use of relative signals makes the sensor quite resistant to changes in environmental conditions such as pressure and temperature differences between calibration and application. This robustness to intrinsic environmental variabilities facilitates the use of the sensor.

Because the sensor is based on a gas transducer, most lander systems equipped with amperometric channels can easily be extended for its use. This enables simultaneous measurements of chemical parameters and flow velocities at high spatial resolution, which helps to gain insight into the processes occurring at the sediment-water interface.

\section{Comments and Recommendation}

Although the relative signals are quite insensitive to changes in the environment, we recommend performing calibrations as close as possible to field conditions. Monitoring flow conditions in the field with additional devices to determine flow direction and vertical flow velocities is also advised, as the sensor is sensitive to vertical velocities. Therefore, it should be used only in systems with negligible vertical components of flow. The sensor could be used to measure flow velocities in systems that have vertical components as well; however, this would require additional calibrations for several inclination angles of the sensor. The direction dependence of the flow sensor demands a simultaneous monitoring of the flow direction. If the direction is constant or changes very slowly, a measurement of the direction at a fixed position is sufficient. If the current directions change very rapidly, the data must be interpreted more carefully. Another critical point is the decrease in sensitivity of the sensor with increasing flow, and large errors have to be taken into account for high velocities. For our sensors, the critical threshold was around $12 \mathrm{~mm} \mathrm{~s}^{-1}$. Under such conditions, thermistors offer a preferable option for flow measurements. The technique introduced here performs best at low velocities. In waters with high particle concentrations, particles may adhere to the sensor tip and lead to increased signals and therefore to an underestimation of the flow. If the sensor is applied in such systems, a cleaning system should be integrated in the measurement setup.

The strengths of this sensor are the high sensitivity to very low velocities, high spatial resolution, and small tip size. It allowed us to perform a detailed study of the structure of the flow profile in the last few $\mathrm{mm}$ above the sediment. Provided that adequate calibration procedures are developed, the sensor opens opportunities for many more potential applications such as flow measurements in capillaries or porous media.

\section{References}

Agrawal, Y. C., and C. J. Belting 1988. Laser velocimetry for benthic sediment transport. Deep-Sea Res. 35:1047-1067.

Bertucciolli, L., G. I. Roth, J. Katz, and T.R. Osborn 1999. A submersible particle image velocimetry system for turbulence measurements in the bottom boundary layer. J. Atmos. Oceanic Tech. 16:1635-1646.

Boudreau, B. P., and N. L. J. Guinasso 1982. The influence of a diffusive sublayer on accretion, dissolution, and diagenesis at the sea floor. In K. A. Fanning and F. T. Manheim [eds.], The Dynamic Environment at the Ocean Floor. University of Miami, p.115-145.

and B. B. Jørgensen, eds. 2001. The Benthic Boundary Layer. Oxford University Press.

Bronstein, I. N., K. A. Semendjajew, G. Musiol, and H. Mühlig. 1997. Taschenbuch der Mathematik. Verlag Harri Deutsch.

Caldwell, D. R., and T. M. Chriss 1979. Viscous sublayer at the sea-floor. Science 205:1131-1132.

Chriss, T. M., and D. R. Caldwell 1982. Evidence for the influence of form drag on bottom boundary-layer flow. J. Geophys. Res. 87:4148-4154.

— and D. R. Caldwell 1984a. Turbulence spectra from the viscous sublayer and buffer layer at the ocean-floor. J. Fluid Mech. 142:39-55.

and D. R. Caldwell 1984b. Universal similarity and the thickness of the viscous sublayer at the ocean-floor. J. Geophys. Res.-Oceans 89:6403-6414.

Crank, J. 1983. The Mathematics of Diffusion. Clarendon Press.

Dade, W. B., A. J. Hogg, and B. P. Boudreau. 2001. Physics of flow above the sediment water interface. In B. P. Boudreau and B. B. Jørgensen [eds.], The Benthic Boundary Layer. Oxford University Press, p. 4-43.

Gloor, M., A. Wüest, and M. Münnich. 1994. Benthic boundary mixing and resuspension induced by internal seiches. Hydrobiol. 284:59-68.

Gust, G. 1988. Skin friction probes for field applications. J. Geophys. Res. 93:14121-14132. 
Jørgensen, B. B., and D. J. D. Marais. 1990. The diffusive boundary-layer of sediments: oxygen microgradients over a microbial mat. Limnol. Oceanogr. 35:1343-1355.

and N. P. Revsbech. 1985. Diffusive boundary-layers and the oxygen-uptake of sediments and detritus. Limnol. Oceanogr. 30:111-122.

Khalili, A., M. Huettel, and W. Merzkirch 2001. Fine-scale flow measurements in the benthic boundary layer. In B. P. Boudreau and B. B. Jørgensen [eds.], The Benthic Boundary Layer. Oxford University Press, p. 44-77.

Lorke, A., B. Müller, M. Maerki, and A. Wüest 2003. Breathing sediments: the control of diffusive transport across the sediment-water interface by periodic boundary layer turbulence. Limnol. Oceanogr. 48:2077-2085.

Müller, B., M. Märki, C. Dinkel, R. Stierli, and B. Wehrli 2002. In situ measurements in lake sediments using ion-selective electrodes with a profiling lander system. In M. Taillefert, T. F. Rozan, [eds], Environmental Electrochemistry: Analyses of Trace Element Biogeochemistry. American Chemical Society, p. 126-143.

Nelson, J. M., R. L. Shreve, S. R. McLean, and T. G. Drake.
1995. Role of near bed turbulence structure in bead load transport and bed form mechanics. Water Res. Res. 31: 2071-2086.

Nimmo Smith, W. A. M., P. Atsavapranee, J. Katz, and T. R. Osborn. 2002. PIV measurements in the bottom boundary layer of the coastal ocean. Exp. Fluids 33:962-971.

- J. Katz, and T. R. Osborn. 2005. On the structure of turbulence in the bottom boundary layer of the coastal ocean. J. Phys. Oceanogr. 35:72-93.

Revsbech, N. P., L. P. Nielsen, and R.B. Ramsing 1998. A novel microsensor for determination of apparent diffusivity in sediments. Limnol. Oceanogr. 43:986-992.

Røy, H., M. Hüttel, and B. B. Jørgensen 2002. The role of small-scale sediment topography for oxygen flux across the diffusive boundary layer. Limnol. Oceanogr. 47: 837-847.

Wüest, A., and A. Lorke 2003. Small-scale hydrodynamics in lakes. Ann. Rev. Fluid Mech. 35:373-412.

Submitted 2 May 2006 Revised 28 November 2006 Accepted 15 December 2006 